\title{
Micobacteriosis atípica de distribución esporotricoide
}

\section{Atypical mycobacterial infections with sporotrichoid distribution}

\author{
Melanie Reichardt ${ }^{1}$, Jesica Radonich ${ }^{2}$, Martina Buffetti ${ }^{1}$, Graciela Carabajal ${ }^{3}$ y Miguel A. Mazzini ${ }^{4}$
}

\section{RESUMEN}

Las micobacterias atípicas o no tuberculosas son un conjunto de especies integrado por más de 150 variedades. Se caracterizan por su amplia distribución en la naturaleza. En ocasiones, resultan patógenas para el ser humano y pueden producir desde afectación pulmonar, linfadenitis, infección de catéteres y enfermedad diseminada hasta infecciones de la piel y los tejidos blandos. Se presentan los casos de dos pacientes con infecciones cutáneas causadas por Mycobacterium

marinum y Mycobacterium chelonae, ambos con clínica de distribución esporotricoide, con buena respuesta al tratamiento instaurado.

Palabras clave: micobacterias atípicas, granuloma de las piscinas, Mycobacterium marinum, Mycobacterium chelonae.

Dermatol. Argent. 2020, 26 (3): 105-109
\end{abstract}

\section{ABSTRACT \\ Nontuberculous mycobacteria are a group of over 150 bacterial species. All of these are environmental organisms and can cause human infection such as pulmonary disease, lymphadenitis, catheter-related infections, disseminated disease or cutaneous and soft tissues infections. Two cases of nontuberculous mycobacteria cutaneous infection involving Mycobacterium marinum and Mycobacterium chelonae both cases with \\ sporotrichoid distribution as clinical manifestation and good response to treatment are presented. \\ Key words: atypical mycobacteria, swimming pool granuloma, Mycobacterium marinum, Mycobacterium chelonae. \\ Dermatol. Argent. 2020, 26 (3): 105-109}

1 Médica Residente de Dermatología

2 Médica Dermatóloga, Jefa de Residentes de Dermatología

${ }^{3}$ Médica Especialista en Anatomía Patológica, Sección Dermatopatología

4 Jefe del Servicio de Dermatología

Servicio de Dermatología, Hospital Churruca-Visca, Ciudad

Autónoma de Buenos Aires, Argentina
Contacto del autor: Melanie Reichardt E-mail: reichardt.mel@gmail.com Fecha de trabajo recibido: 25/5/2020

Fecha de trabajo aceptado: 30/7/2020

Conflicto de interés: los autores declaran que no existe conflicto de interés.

\section{INTRODUCCIÓN}

El descubrimiento, en 1882, por parte del médico y microbiólogo Robert Koch, del patógeno causante de la tuberculosis es conocido como el evento fundamental que permitió abrir el abanico en el estudio de las especies dentro del género Mycobacterium. Durante muchos años se estudiaron las características distintivas de un porcentaje de estas con respecto al complejo $M$. tuberculosis. Finalmente, en 1959, Ernest H. Runyon las caracterizó y agrupó en la clasificación que lleva su nombre. Para ello, utilizó como criterios en su división la velocidad de crecimiento y la presencia, o no, de pigmento $^{1,2}$. En la clasificación mencionada, los grupos I a III corresponden a micobacterias de crecimiento lento (más de 7 días). Las del grupo I son fotocromógenas (producen pigmento ante la exposición a la luz), las del grupo II son escotocromógenas (producen pigmento con exposición o no a la luz) y las del grupo III son no cromógenas (no producen pigmento). El grupo 
IV corresponde a micobacterias de crecimiento rápido (menos de 7 días) (Tabla 1).

Un gran porcentaje de las micobacterias no tuberculosas patógenas pueden causar una infección cutánea. Las especies de crecimiento rápido ( $M$. fortuitum, M. chelonae), $M$. marinum y $M$. ulcerans son las involucradas con mayor frecuencia y casi todas tienen distribución mundial ${ }^{3}$.

Se comunican los casos de dos pacientes evaluados en nuestro servicio con nódulos de distribución lineal que parecían seguir los trayectos linfáticos. Se analizan la clínica, el diagnóstico y el tratamiento de esta patología. Destacamos la importancia del rescate bacteriológico para el correcto diagnóstico y oportuno tratamiento.

\begin{tabular}{|l|c|}
\hline \multicolumn{1}{|c|}{ Especie } & $\begin{array}{c}\text { Grupo de } \\
\text { Runyon }\end{array}$ \\
\hline M. kansasii, M. marinum & I \\
\hline M, scrofulaceum, M. szulgai, M. gordonae & II \\
\hline M. avium-intracellulare, M. haemophilum, M. ulcerans & III \\
\hline M. fortuitum, M. chelonae, M. abscessus & IV \\
\hline $\begin{array}{l}\text { TABLA 1: Clasificación de Runyon de las micobacterias atípicas que las divide } \\
\text { en cuatro grupos. }\end{array}$
\end{tabular}

\section{CASO CLÍNICO 1}

Una mujer de 71 años, con antecedentes de artrosis en la rodilla y colon irritable sin tratamiento médico, consultó por presentar una dermatosis localizada en el antebrazo izquierdo, de 2 meses de evolución.

Presentaba múltiples lesiones nodulares levemente dolorosas distribuidas en la cara externa del antebrazo izquierdo, de manera lineal sobre el trayecto linfático (Foto 1). En la palpación tenían consistencia firme y móvil, sin adherencia a los planos profundos. La piel suprayacente no mostraba cambios. Según refirió la paciente, las lesiones comenzaron después de realizar una actividad de buceo en Australia. El cuadro cutáneo no se acompañaba de sintomatología sistémica.

Se realizó una biopsia de piel para anatomía patológica y se tomaron muestras para cultivo de micobacterias y hongos. En la anatomía patológica, se evidenció una epidermis sin alteraciones y la presencia de granulomas sarcoidales en la dermis reticular profunda y la hipodermis. Mediante las técnicas de coloración PAS y Grocott no se identificaron elementos micóticos. Con la tinción de Ziehl-Neelsen no se observaron bacilos ácido-alcohol resistentes (BAAR) (Foto 2). De manera diferida, se obtuvo un cultivo positivo para Mycobacterium marinum. Con los antecedentes epidemiológicos, la clínica y los exámenes complementarios, arribamos al diagnóstico de micobacteriosis atípica por M. marinum.

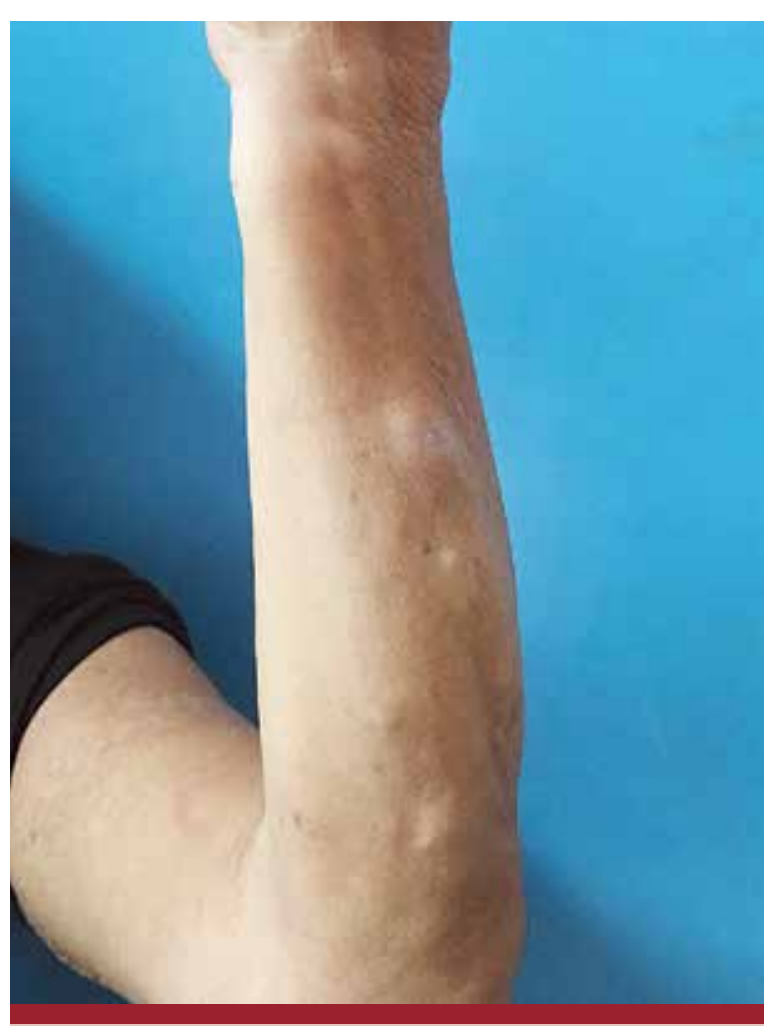

FOTO 1: Múltiples lesiones nodulares distribuidas en la cara externa del antebrazo izquierdo que siguen el trayecto linfático.

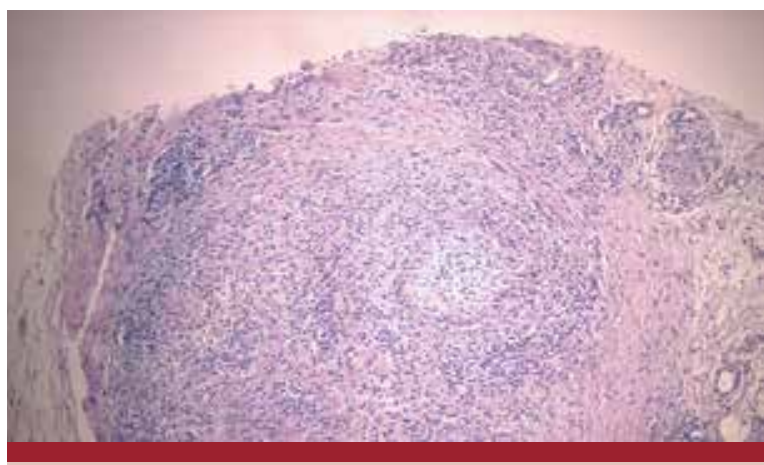

FOTO 2: Granulomas sarcoidales en la dermis reticular profunda y en la hipodermis (HyE, 10X).

Se indicó tratamiento combinado con rifampicina $600 \mathrm{mg} /$ día, claritromicina $500 \mathrm{mg}$ cada 12 horas y ciprofloxacina $500 \mathrm{mg}$ cada 12 horas.

Hasta la publicación de este trabajo, la paciente había cumplido 3 meses de tratamiento, con respuesta parcial y disminución del número y el tamańo de los nódulos. Se le indicó continuar con el mismo esquema terapéutico hasta la resolución total de las lesiones.

\section{CASO CLIÍNICO 2}

Corresponde a una mujer de 75 años, con antecedentes de hipertensión arterial, diabetes tipo 2, carcinoma papilar de tiroides con tratamiento quirúrgico y 
posterior hipotiroidismo, artritis reumatoide (en tratamiento con metotrexato los últimos 2 ańos) y vasculitis p-ANCA (poliangitis microscópica). Esta última presentaba compromiso cutáneo y pulmonar, por lo que se le indicaron pulsos de metilprednisolona y ciclofosfamida 4 meses antes de la consulta.

Durante el control reumatológico, se evidenciaban lesiones en la piel de aspecto nodular, distribuidas en el miembro inferior izquierdo, que motivaron al reumatólogo a solicitar una evaluación dermatológica.

En el examen físico, se observaron lesiones nodulares parduzcas, móviles a la palpación, algunas de las cuales habían drenado su contenido al exterior (gomas) y eran asintomáticas. Se distribuían unilateralmente y parecían seguir el trayecto linfático (Foto 3). Por la distribución de las lesiones y el contexto de la inmunosupresión, se planteó la sospecha diagnóstica de micobacteriosis atípica. Tras realizar un exhaustivo interrogatorio dirigido, no se determinó ningún antecedente de exposición a posibles fuentes de adquisición de micobacterias.

Se tomó una biopsia incisional de una de las gomas ubicadas en la cara interna del muslo izquierdo para cultivo. En el examen directo se evidenciaron múltiples BAAR. A las 7 semanas se obtuvo la tipificación de Mycobacterium chelonae en el cultivo.

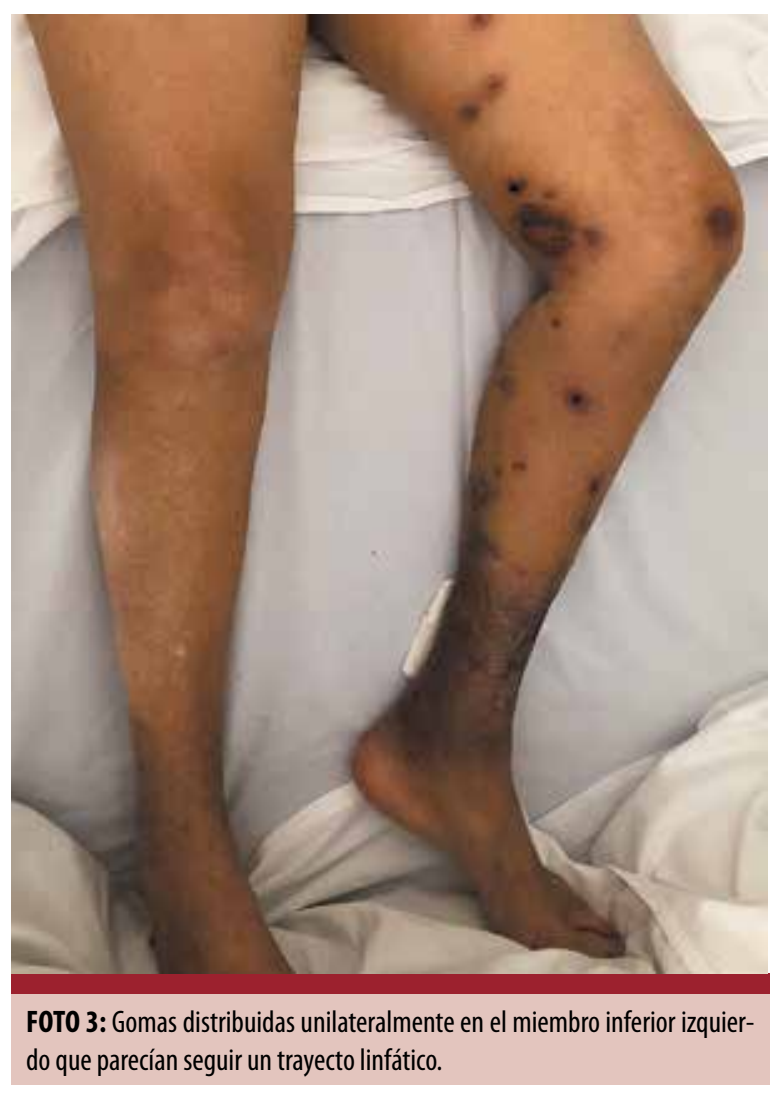

Se indicó tratamiento con levofloxacina $750 \mathrm{mg}$ por día y claritromicina $500 \mathrm{mg}$ dos veces por día. La paciente interrumpió el seguimiento en nuestro servicio, por lo que no se pudo evaluar su evolución dermatológica.

A los 5 meses de iniciado el tratamiento, la paciente fue internada en la unidad de terapia intensiva por una sepsis con foco urinario y aislamiento de Klebsiella pneumoniae. Los familiares a cargo refirieron que la paciente, hasta ese momento, no había discontinuado el tratamiento antibiótico indicado. En el examen físico, presentaba franca mejoría de las lesiones cutáneas y se evidenciaban solo máculas hiperpigmentadas residuales (Foto 4). Si bien tuvo una excelente evolución del cuadro cutáneo, el cuadro séptico de foco urinario progresó a shock séptico y la paciente murió a los 10 días. Cabe aclarar que no se halló ninguna relación entre la urosepsis que le causó la muerte y la micobacteriosis cutánea.

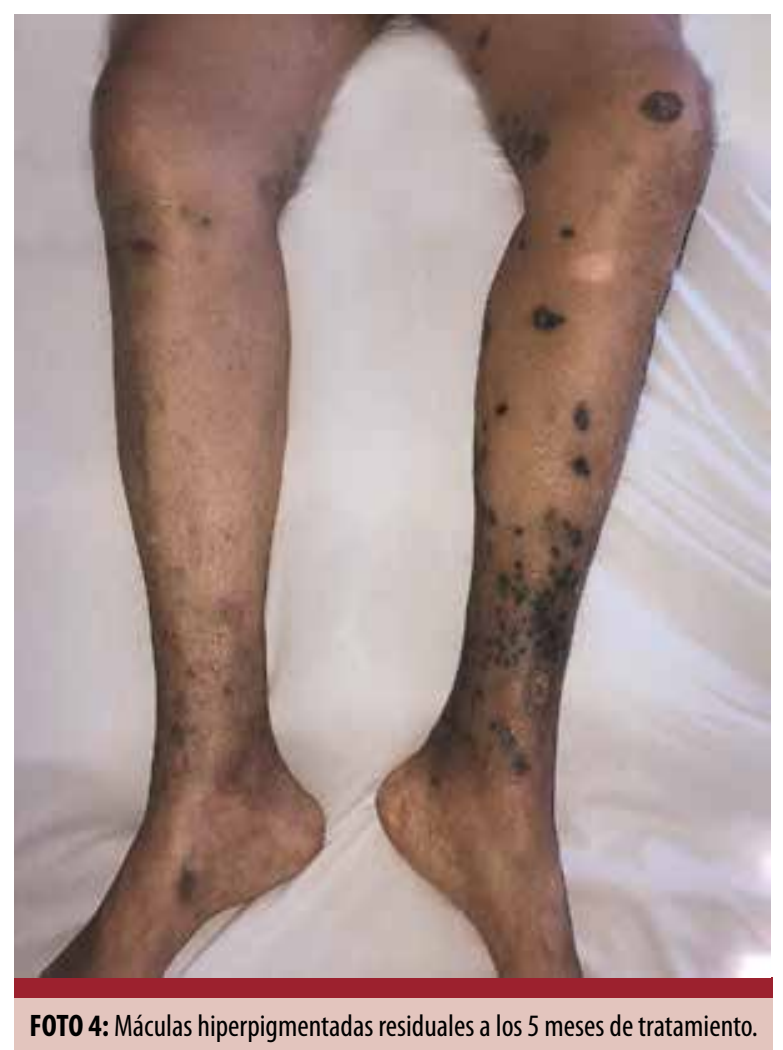

\section{COMENTARIOS}

Las bacterias del género Mycobacterium se caracterizan por ser bacilos ácido-alcohol resistentes aerobios e inmóviles, que no forman esporas y tienen un alto contenido de lípidos en la pared celular. Esta característica les otorga baja permeabilidad a las soluciones colorantes, el agua o los agentes microbicidas, lo que permite su supervivencia en diferentes ambientes ${ }^{4}$. 
Según las características nosológicas, las micobacterias pueden reunirse en tres grupos ${ }^{2}$ :

- Complejo M. tuberculosis, compuesto por especies de $M$. tuberculosis, M. bovis, M. africanum y $M$. microti, especies causantes de la enfermedad infecciosa bacteriana conocida como tuberculosis.

- M. leprae y M. lepromatosis, especies causantes de la enfermedad infecciosa crónica conocida como lepra.

- Micobacterias no tuberculosas (MNT) o micobacterias atípicas, que presentan características microbiológicas distintas de las mencionadas con respecto a la velocidad de crecimiento o la presencia o no de pigmento.

Debido a los avances de la bacteriología hacia finales del siglo pasado, se ha logrado tipificar distintas MNT causantes de diversos cuadros infecciosos 5 . Se describieron alrededor de 150 especies y aproximadamente la mitad de ellas se consideran posibles patógenos para el ser humano, causantes de un espectro de manifestaciones clínicas denominadas micobacteriosis $^{3}$. Las especies involucradas con mayor asiduidad son las de crecimiento rápido $(M$. fortuitum, $M$. chelonae, M. abscessus), M. marinum y M. ulcerans 3 .

Estos microorganismos, que se encuentran distribuidos en la naturaleza, pueden aislarse de fuentes naturales de agua (ríos, lagos, pantanos) y también de fuentes artificiales (piscinas, acuarios, máquinas de hielo). A su vez, pueden hallarse en el suelo y la vegetación, así como en algunos animales, principalmente peces y corales ${ }^{6}$. Algunas de las actividades que propician su adquisición son las acuáticas como el buceo y el esnórquel y las recreativas en los acuarios. También se han informado numerosos casos de micobacteriosis atípicas provocadas por procedimientos estéticos en pacientes jóvenes inmunocompetentes debido a dos factores claves: por un lado, la ubicuidad de estos microorganismos, por lo que pueden colonizar tanto el instrumental como las soluciones utilizadas en esos procedimientos y, por otro lado, el microtraumatismo, que funcionaría como la puerta de entrada ${ }^{6}$.

El principal factor de riesgo para adquirir esta enfermedad es la inmunosupresión. La mayoría de los casos se describen en pacientes con infecciones crónicas, patología oncológica o terapia inmunosupresora ${ }^{7}$. Tanto el HIV como la terapia antirretroviral han contribuido al incremento en las infecciones por $\mathrm{MNT}^{1}$. Los principales factores de riesgo para contraer una enfermedad cutánea por $M$. chelonae son los traumatismos previos y los tratamientos prolongados con corticosteroides. Se hace especial hincapié en los pacientes con enfermedades autoinmunes que requieren corticoterapia sistémica prolongada, como ocurría en el caso clínico 2. A su vez, se describieron como trastornos concomitantes la artritis reumatoide, los trasplantes y la hemodiálisis ${ }^{1}$. Algunas revisiones indican que aproximadamente la mitad de los pacientes diagnosticados con infecciones causadas por estos agentes se encontraban en tratamiento corticosteroideo ${ }^{8}$. En el caso de M. marinum, se considera como principal factor de riesgo el antecedente de exposición a fuentes de contagio características como acuarios, estanques, piscinas o peces tropicales, tras sufrir pequeños traumatismos cutáneos $^{3}$. Esta última fue la fuente de contagio identificada en la paciente del caso 1 .

El espectro de formas clínicas en las que se presentan las MNT es amplio. Puede abarcar desde afectación pulmonar, linfadenitis, infección de catéteres y enfermedad diseminada hasta infecciones de la piel y los tejidos blandos.

En lo que respecta a la afectación cutánea, puede presentarse como pápulas, placas, nódulos, abscesos o gomas ${ }^{1}$. En la mayoría de los casos se evidencia una lesión única; sin embargo, algunas especies adoptan ciertos patrones morfológicos, como el esporotricoide, evidenciado en los pacientes presentados. Tal es el caso de M. marinum, M. kansasii y M. chelonae. En algunas publicaciones, la distribución esporotricoide se describe como la forma de presentación más frecuente de $M . m a-$ rinum cuando la dermatosis se contrae en los acuarios ${ }^{1}$.

Las manifestaciones histopatológicas con tinción de hematoxilina y eosina son muy heterogéneas, dado que las diversas presentaciones clínicas dependen del estado inmunitario del paciente y del tiempo de evolución de la dermatosis. En los pacientes inmunodeprimidos, se evidencia un infiltrado inflamatorio difuso en la dermis profunda y en la hipodermis. En cambio, en los inmunocompetentes, la inflamación suele afectar la dermis profunda con formación de granulomas en el $80 \%$ de los casos ${ }^{1}$. A su vez, se describieron ciertas características histológicas específicas según la especie implicada. En M. marinum, los cambios histológicos se dividen en una fase aguda caracterizada por un infiltrado linfohistiocitario inespecífico y una fase crónica en las lesiones de más de 3 meses de evolución, donde ya se evidencian granulomas ${ }^{9}$. Dentro de los primeros 6 meses de la dermatosis, los granulomas pueden ser de tipo sarcoidal o desnudo (como se observó en el caso clínico 1). Pasados los 6 meses, adquieren la morfología de los granulomas tuberculoides típicos de las micobacterias ${ }^{10}$.

La observación directa de BAAR en el microscopio óptico con tinción de Ziehl-Neelsen constituye el método más sencillo y económico de diagnóstico. No obstante, se considera de baja sensibilidad, ya que se hallan bacilos solo en el 15\% de los casos ${ }^{7}$. 
El cultivo en el medio de Lowenstein-Jensen y en otros caldos de cultivo es la prueba de referencia para el diagnóstico de las $\mathrm{MNT}^{2}$. Sin embargo, su rédito está en relación directamente proporcional a la cantidad de bacilos en la muestra obtenida. La necesidad de obtener un gran inóculo, sumada a la demora en el crecimiento de estos gérmenes, lo hace un método diagnóstico difícil de implementar ${ }^{1}$.

Si bien la reacción en cadena de la polimerasa (PCR) es un método molecular de alta sensibilidad y especificidad, debe acompañarse de otros métodos positivos, como el cultivo o la histopatología, para confirmar el diagnóstico ${ }^{1}$.

Los tratamientos contra las infecciones por MNT incluyen la antibioticoterapia y una serie de procedimientos quirúrgicos. En líneas generales, se aconseja la utilización de dos fármacos durante al menos 2 meses después de la desaparición de los síntomas. Las micobacterias son sensibles a claritromicina, rifampicina, etambutol, tetraciclinas y cotrimoxazol ${ }^{3}$. La importancia de adoptar un esquema multifarmacológico radica en evitar el desarrollo de resistencia antibiótica a los

\section{BIBLIOGRAFÍA}

1. Valdés F, Cid A. Micobacterias atípicas: revisión. Actas Dermosifiliogr 2004;95:331-357.

2. Mancheno-Valencia MA, Arenas-Guzmán R, Carrillo-Casas EM, Fernández-Martínez R, et ál. La infección por micobacterias no tuberculosas, una visión desde la perspectiva dermatológica. Med Cutan Iber Lat Am. 2015;43:S6-S13.

3. Alcaide F, Esteban J. Infecciones cutáneas y de partes blandas por micobacterias no tuberculosas. Enferm Infecc Mibrobiol Clin 2010;28:46-50.

4. Rizzo Gnatta J, Fumiko Sato Kurebayashi L, Paes da Silva MJ. Micobacterias atípicas asociadas a la acupuntura: revisión integral. Rev Latinoam Enfermagen 2013;21:450-458.

5. Casal MM, Casal M. Las micobacterias atípicas como patógenos emergentes. EnfEmerg 2000;2:220-230.

6. Hunt $C$, Olivares $L$, Jaled $M$, Cergneux $F$, et ál. Infección por Mycobacterium marinum: a propósito de tres casos. Dermatol Argent 2013;19:332-336. macrólidos, ya que este grupo de fármacos representan la base de todos los tratamientos contra las MNT. La preferencia por los macrólidos se basa en que estos tienen la mejor relación de comportamiento in vitrol in vivo ${ }^{11}$. No obstante, se aconseja corroborar la sensibilidad antibiótica del aislamiento microbiológico siempre que sea posible.

El desbridamiento quirúrgico anterior a la antibioticoterapia se recomienda en las infecciones por M. fortuitum y $M$. chelonae, tras haberse demostrado resultados favorables con el uso de esta combinación terapéutica ${ }^{7}$.

Se presentan dos casos de micobacteriosis atípica, un trastorno de baja frecuencia en nuestro medio, que suele afectar a pacientes inmunosuprimidos. Esta afirmación no se cumple en el primer caso comentado, en el que no se hallaron antecedentes médicos de inmunosupresión, pero sí en el segundo caso. Consideramos que, ante lesiones clínicas sospechosas, aun en pacientes inmunocompetentes, se deben investigar los antecedentes epidemiológicos para arribar al diagnóstico de esta patología.

7. Jaramillo-Manzur SC, Medina-Castillo DE, Pérez-López JA Torres-Valencia OG, et ál. Úlcera crónica por Mycobacterium chelonae: informe de dos casos y claves para el diagnóstico de micobacteriosis cutáneas. Med Int Mex 2018;34:985-993.

8. Lamb RC, Dawn G. Cutaneous non-tuberculous mycobacterial infections. Int J Dermatol 2014;53:1197-1204.

9. Ruiz SM, Medina Castillo D, Vences M, Vidal FA. Granuloma de las piscinas de distribución esporotricoide. Rev Cent Dermatol Pascua 2002;11:150-152.

10. Del Solar M, Salomón M, Bravo F, Seas $F$, et ál. Infección cutánea por micobacterias atípicas de crecimiento rápido (MACR) debido a mesoterapia cosmética. Folia dermatol Peru 2005; 16:127-135.

11. Bento CM, Gomes MS, Silva T. Looking beyond typical treatments for atypical mycobacteria. Antibiotics (Basel) 2020;9:3-25. 